\title{
Commentary: Humpty Dumpty and creative autotransplantation
}

\author{
Benjamin D. Kozower, MD, MPH
}

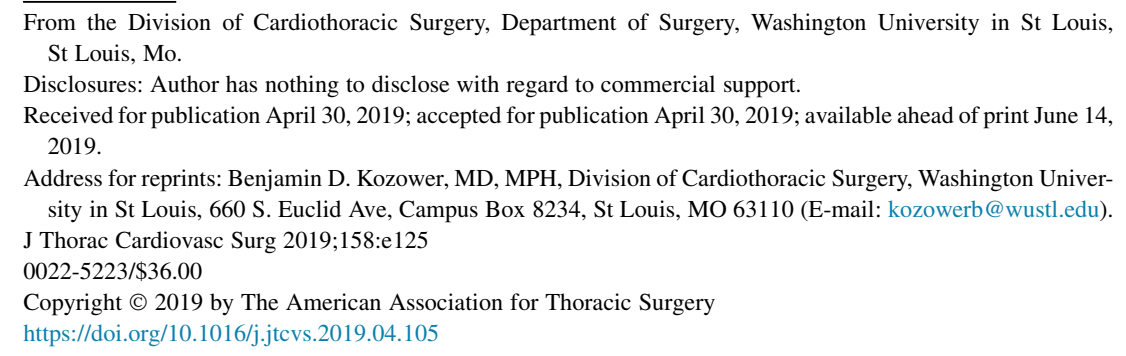

Hamaji and colleagues ${ }^{1}$ describe a creative solution to a difficult postoperative complication. A 55-year-old man presented 2 months postoperatively with an anastomotic stricture after a right upper sleeve lobectomy for stage II squamous cell carcinoma. The surgical team appropriately attempted bronchoscopic intervention, but dilation and argon plasma coagulation were unsuccessful in opening up the middle and lower lobes. After proceeding with additional debridement, a bronchopleural fistula developed at the orifice of the right middle lobe.

The stricture was likely secondary to ischemia of the anastomosis resulting from an aggressive and appropriate nodal dissection for the initial stage II non-small cell lung cancer. Reoperation with the intent of repairing the fistula or revising the sleeve anastomosis is unlikely to result in a satisfactory outcome, and a completion pneumonectomy is likely. This team also has an outstanding lung transplantation program that facilitated this completion pneumonectomy, back-table right middle lobectomy, and right lower lobe/right main-stem anastomosis.

Lung transplantation was successfully performed and brought into clinical practice in Toronto. ${ }^{2}$ The Toronto group also had a busy thoracic oncology practice. The senior author of this report, Dr Hiroshi Date, trained with Drs Joel Cooper and Alec Patterson, and moved from Toronto to St Louis before starting his own highly successful thoracic surgery program in Japan. This is important to note, because there is tremendous benefit from crosstraining in the development of such creativity and technical expertise. The current trend in North America has been to separate lung transplantation from thoracic oncology programs. Dedicated transplant surgeons who do not routinely perform general thoracic surgery lead many high-volume transplant programs.

This case report illustrates a risky and creative solution to a difficult postoperative complication. If endoscopic management were unsuccessful, the traditional solution for autotransplantation.

\section{References} e121-3. 184-95.

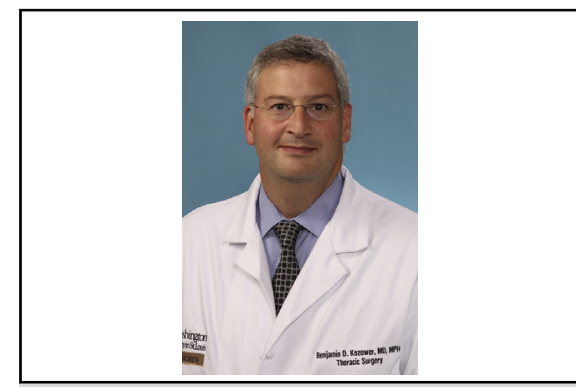

Benjamin D. Kozower, MD, MPH

Central Message

This report details a creative management strategy for a postresection bronchopleural fistula. The authors used an autotransplantation or ex situ reconstruction method to perform a difficult revision sleeve resection after a right upper sleeve lobectomy with stricture and fistula.

See Article page e121.

this post-sleeve resection stricture/fistula would be a completion pneumonectomy. This is probably the safest management strategy for the community of thoracic surgeons performing sleeve lobectomies. However, this has the highest morbidity and mortality rate of any lung resection and leaves a contaminated pleural space to manage. ${ }^{3}$ If a patient will not tolerate a pneumonectomy at the time of the initial surgery because of limited pulmonary function, surgeons need to be very thoughtful before attempting a sleeve lobectomy, which may not be possible intraoperatively. Fortunately, this patient was cared for by a thoracic unit experienced in both lung transplantation and thoracic oncology. They had the creativity and technical expertise to combine their resources and perform this successful

1. Hamaji M, Chen-Yoshikawa TF, Date H. Completion pneumonectomy and autotransplantation for bronchopleural fistula. J Thorac Cardiovasc Surg. 2019;158:

2. Toronto Lung Transplant Group. Unilateral lung transplantation for pulmonary fibrosis. N Engl J Med. 1986;314:1140-5.

3. Pagès PB, Mordant P, Renaud S, Brouchet L, Thomas PA, Dahan M, et al. Sleeve lobectomy may provide better outcomes than pneumonectomy for non-small cell lung cancer: a decade in a nationwide study. J Thorac Cardiovasc Surg. 2017;153: 